\title{
Recurrent anuria- A Rare Presentation of a Gommon Entity
}

Sandeep Gupta, Khumukcham Somarendra, Akoijam Kaku Singh, Sinam Rajendra Singh From the Department of Urology, Regional Institute of Medical Sciences, Imphal-795004, India.

\section{Abstract:}

Anuria is defined as less than $50 \mathrm{cc}$ of urine output in 24 hours. Obstruction to the flow of urine on both the sides and obstructive uropathy in a solitary kidney can lead to this condition. Complete absence of one kidney occurs more frequently than bilateral renal agenesis but is not easily detected from findings on physical examination. Unilateral renal agenesis may remain undetected unless examination of the external genitalia and/or radiographic evaluation of the female or male pelvis for other reasons reveal an anomaly associated with renal agenesis. As in this case, the entity came to light only when the patient presented to us with anuria due to pelvi-ureteric junction obstruction (PUJO) with renal calculi. It was only after he had recurrent anuria following $D$-J stent removal that an on table retro-grade pyelography was able to establish the PUJO. Such cases need to be regularly followed-up to monitor for any signs of renal failure. PUJO presenting as recurrent anuria per se is an uncommon mode of presentation.

Key words: Anuria, Urogenital Abnormalities, Multicystic Dysplastic Kidney, Kidney Diseases.

\section{Introduction}

Obstruction of the urinary tract can occur during fetal development, childhood, or adulthood. The point of obstruction can be from calyces to urethral meatus. Pelvi-ureteric junction obstruction (henceforth PUJO) is a partial or total blockage of the flow of urine that occurs where the ureter enters the kidney. The aetiology of PUJ obstruction includes both congenital and acquired conditions. PUJ obstruction is the most common pathologic cause of antenatally detected hydronephrosis. Most of the causes of PUJ obstruction are congenital but from time to time urologist will come across cases of secondary PUJ obstruction related to surgery, infection or trauma.
The diagnosis of "ureteropelvic junction obstruction" results in a functionally significant impairment of urinary transport from the renal pelvis to the ureter. Although most cases are congenital, the problem may not become clinically apparent until much later in life. Acquired conditions such as stone disease, postoperative or inflammatory stricture, or urothelial neoplasm may also present clinically with symptoms and signs of obstruction at the level of the ureteropelvic junction (PUJ). This entity per se is a common one but we are highlighting this case in view of unusual mode of presentation of PUJO in a solitary kidney.

\section{Corresponding Author: Dr. Sandeep Gupta}

Email: drsandeepgupta2009@yahoo.in

Received: September 8, 2013 | Accepted: September 26, 2013 | Published Online: October 25, 2013 This is an Open Access article distributed under the terms of the Creative Commons Attribution License (creativecommons.org/licenses/by/3.0)

Conflict of interest: None declared | Source of funding: Nil | DOl: http://dx.doi.org/10.17659/01.2013.0088 


\section{Case Report}

A 27 year old male presented to us with anuria for the last 2 days associated with fever and vomiting. Examination revealed fullness and tenderness of the left flank with nonpalpable urinary bladder. Screening ultrasound revealed left sided gross hydronephrosis with a two calculi measuring around $11 \mathrm{~mm}$ in the lower calyx along with non-visualisation of right kidney and empty urinary bladder. He did not give any history of surgeries in the past. His serum creatinine was 3.8 $\mathrm{mg} \%$. An emergency left sided double-J stenting was done. On cystoscopy, the right ureteric orifice was not visualised. Following this, his urine output and serum creatinine gradually normalised and the patient underwent non-contrast CT-scan of the KUB region which revealed left renal lower calyceal calculus $(11 \mathrm{~mm})$ with mild hydronephrosis and left D-J in situ [Fig. 1 A]. A small $4 \mathrm{~mm}$ left upper ureteric calculus was also visible adjacent to the D-J stent [Fig.1B]. The right kidney and ureter were not

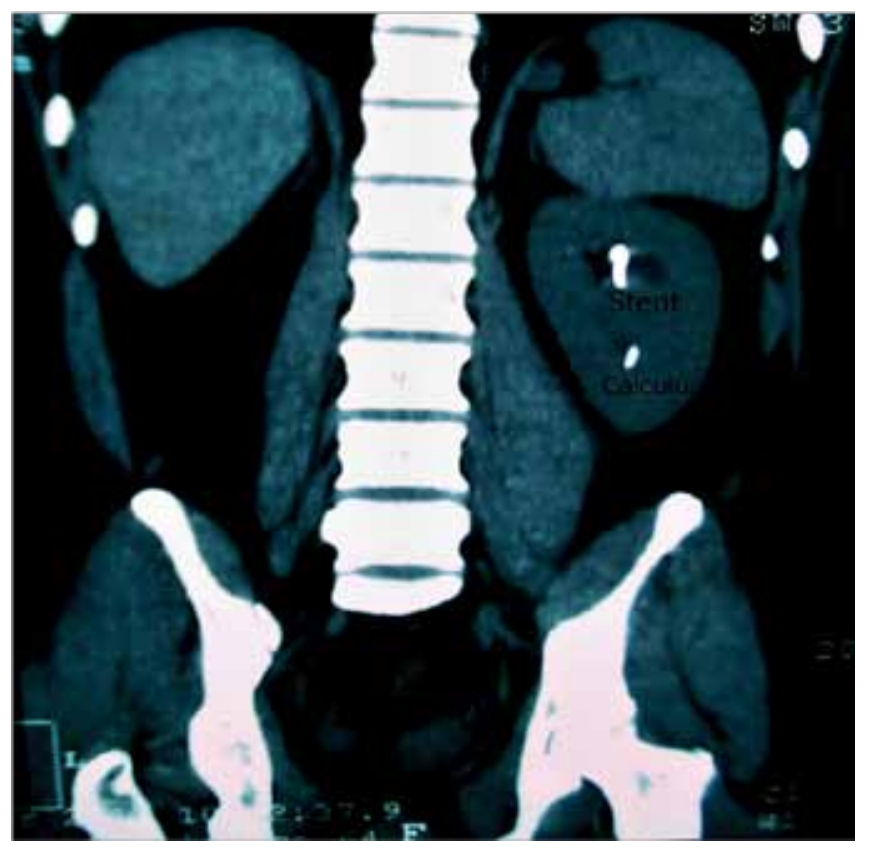

Fig.1 A: NCCT scan of KUB region showing left lower calyceal calculus with left D-J stent. visible in any of the films. The patient was given the various possible treatment options and he decided to undergo Extra-corporeal Shock wave lithotripsy (ESWL). After 3 sessions of ESWL, the X-ray KUB revealed small fragments in the left upper ureter with no evidence of calculus in the kidney. The left D-J stent was subsequently removed and the patient discharged. After about 2 weeks, the patient again presented with anuria following which left D-J stenting was done. Ultrasound screening revealed left gross hydronephrosis with small PUJ calculi with D-J stent in situ. The intravenous pyelography done after normalisation of serum creatinine showed left gross hydronephrosis with dilated renal pelvis [Fig.2A]. It also revealed a left upper ureteric calculus [Fig.2B]. No contrast uptake/excretion was seen on the right side. Based on the IVP findings, 2 more sessions of ESWL were done after which the $X$-ray revealed complete clearance. Hence, the left D-J stent was removed but the patient again went into post procedure retention. The next day, an on table retrograde pyelography (RGP) was planned.

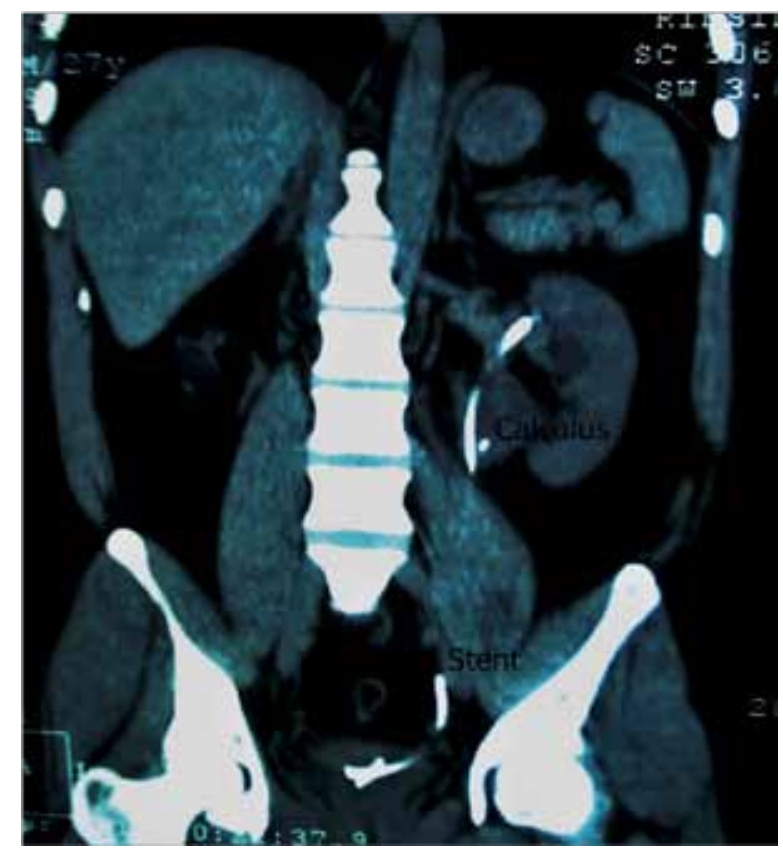

Fig.1B: NCCT scan of KUB region showing left D-J stent with small upper ureteric calculus. 


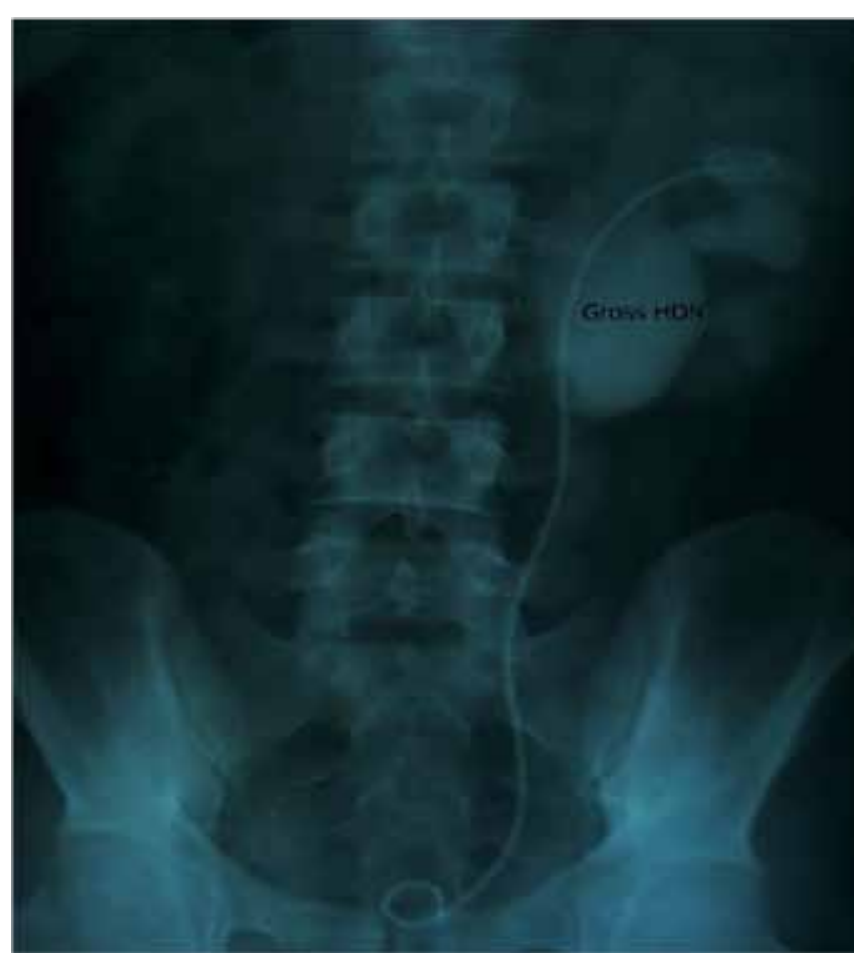

Fig.2A: IVP showing left gross hydronephrosis with dilated renal pelvis.

The RGP revealed evidence of left pelvi-ureteric junction obstruction [Fig.3]. Re-stenting was done and the patient underwent left Anderson-Hyne's dismembered open pyeloplasty at a later date. Post operatively, the D-J stent was removed after six weeks without any further episodes of urinary retention. The patient is on regular follow up and his ultrasound and serum creatinine are within normal limits. Hence, we successfully diagnosed and managed an interesting misleading presentation of a common entity in a solitary kidney.

\section{Discussion}

Unilateral renal agenesis (URA) is a congenital condition that may be caused by Wolffian duct maldevelopment, ureteral bud abnormalities, or degeneration of the ureteral bud at an early stage [1]. It is found in about $0.1 \%$ of autopsies

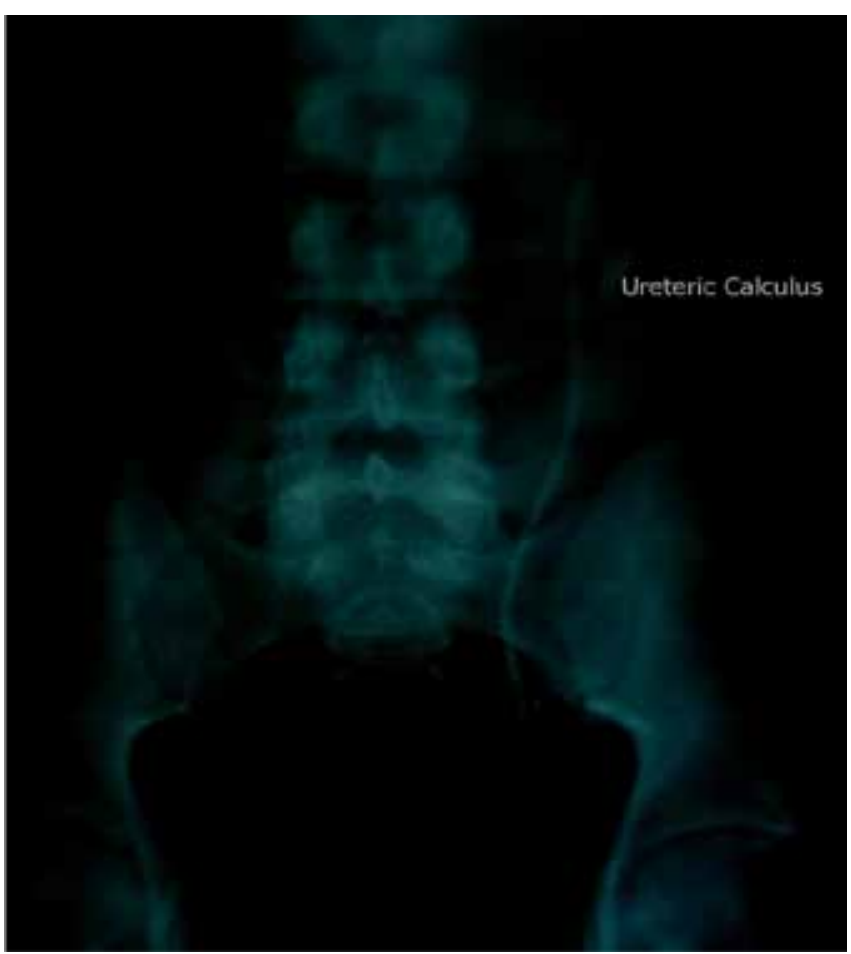

Fig.2B: IVP showing left upper ureteric calculi.

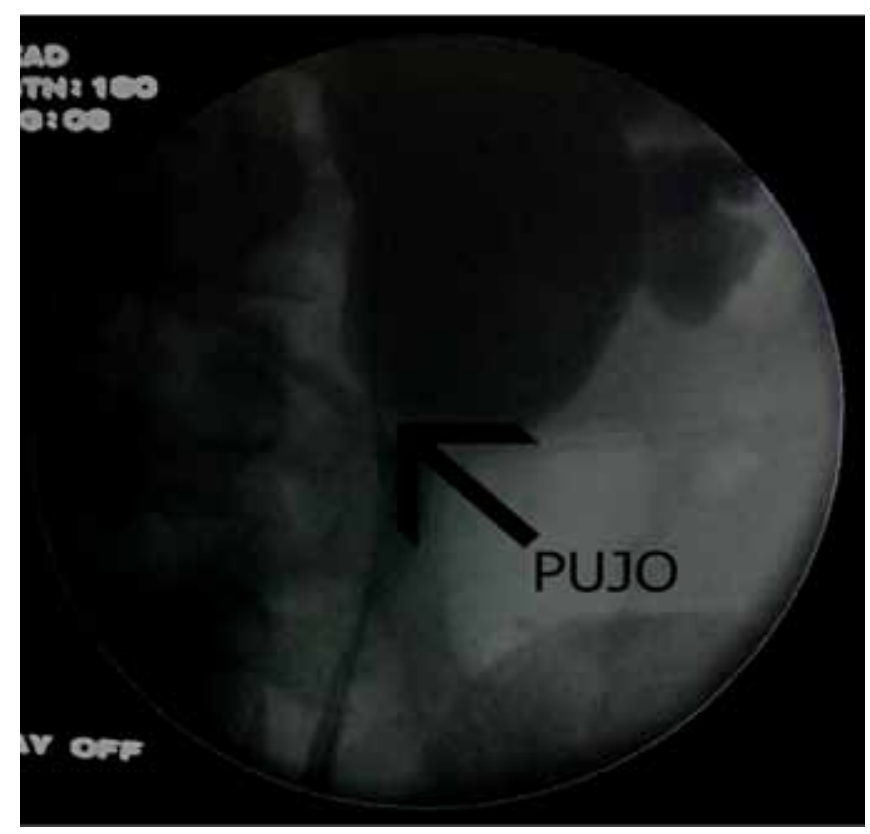

Fig.3: Retrograde Pyelography (RGP) showing the classical PUJ obstruction on the left side. 
and has been associated with other non-urological malformations and contralateral collecting system anomalies [2]. This anomaly is relatively rare, because much of "empty lumbar pits" comparable to agenesis, correspond in fact to the presence of small hyperplasic and/or dysplastic kidney escaping from the conventional means of diagnosis [3]. Prenatal imaging studies have also revealed that a substantial number of cases thought to be URA were dysplastic or multicystic dysplastic kidney (MCDK) that had undergone involution prior to birth. A plain film of the abdomen supports this diagnosis if the splenic or hepatic flexure of the bowel is in its normal location and not in the ipsilateral renal fossa, suggesting that a dysplastic kidney or MCDK may have formed in the renal fossa before involution. URA may remain undetected unless examination of the external genitalia and/or radiographic evaluation of the female or male pelvis for other reasons reveal an anomaly associated with renal agenesis. Except for ectopia or malrotation, anomalies of the contra lateral kidney are very infrequently encountered [4]. However, abnormalities of the contralateral collecting system are not uncommon, including ureteropelvic and ureterovesical junction obstruction in $11 \%$ and $7 \%$, respectively [2].

Absence of a kidney occurs somewhat more frequently on the left side [5]. However, in this case the URA was on the right side. The ipsilateral ureter is completely absent in about $60 \%$ of the cases, as in this case [5]. Pelvi-ureteric junction (PUJ) obstruction is probably the commonest congenital abnormality of the ureter, with a reported incidence of about 5 per 1,00,000 of the population per year [6]. Although most cases of PUJ obstruction are congenital, they may not be manifested until much later in life, as in this case [7]. A less frequent intrinsic cause of congenital UPJ obstruction, as suspected in this case, is true ureteral stricture. Such congenital ureteral strictures are most frequently found at the UPJ, although they may be located at sites anywhere along the lumbar ureter.
Abnormalities of ureteral musculature have been implicated as electron microscopy has demonstrated excessive collagen deposition at the site of the stricture [7]. When acute, complete ureteral obstruction is promptly relieved, full recovery of glomerular filtration (GFR) can occur [8]. Many reports have emphasized on the importance of a complete urologic evaluation of the contra lateral renal tract in cases of URA $[9,10]$. Contemporary indications for intervention for UPJ obstruction include the presence of symptoms associated with the obstruction, impairment of overall renal function or progressive impairment of ipsilateral function, development of stones or infection, or, rarely, causal hypertension [7]. Till date, the Anderson-Hynes dismembered pyeloplasty is considered the surgical repair of choice in PUJO [7]. Such cases should be considered for life-long follow-up by both the nephrologist and the urologist since hypertension, proteinuria, and renal insufficiency have been reported in long-term studies [11-13]. Rovetto et al. [14] have proposed a follow-up protocol of such rare and interesting cases, which we are also using in the follow up of this case. Till date, very few such cases of URA with contra lateral PUJO have been reported in the literature. In one such published data by Thapa et al, they concluded that following successful dismembered pyeloplasty, both their patients did well on long term follow-up [15].

\section{Conclusion}

A case of anuria needs to be thoroughly evaluated to find out the root cause of it, after relieving the patient of his obstruction. Anuria can be a nephrologic or a urological emergency. Prompt and early intervention is the key to renal preservation.

\section{References}

1. Mesrobian H, Rushton H, Bulas D. Unilateral renal agenesis may result from in utero regression of multicystic renal dysplasia. J 
Urol.1993;150:793-794.

2. Cascio S, Paran S, Puri P. Associated urologic anomalies in children with unilateral renal agenesis. J Urol. 1999;162:1081-1083.

3. Ghfir I, Fellah S, Raiis NB. Unilateral renal agenesis revealed by hydronephrosis of contralateral kidney and explored by $99 \mathrm{mTc}$ DMSA and 99mTc-DTPA scintigraphy. The Internet Journal of Nuclear Medicine. 2007;4(2) DOI: 10.5580/10cf.

4. Chow JS, Benson CB, Lebowitz RL. The Clinical Significance of an Empty Renal Fossa on Prenatal Sonography. J Ultrasound Med. 2005;24:1049-1054.

5. Shapiro E, Baver SB, Chow JS. Anomalies of the Upper Urinary Tract. In: Campbell-Walsh. Urology. $10^{\text {th }}$ edition. Saunders: Philadelphia; 2011 . pp. 3123-3160.

6. Eden CG. Treatment options for pelvi-ureteric junction obstruction: implications for practice and training. British Journal of Urology. 1997; 80:365-372.

7. Nakada SY, Thomas H.S. Hsu. Management of Upper Urinary Tract Obstruction. In:CampbellWalsh. Urology. $10^{\text {th }}$ edition. Saunders: Philadelphia; 2011. pp. $1122-1168$.

8. Singh I, Jack W. Strandhoy, Dean G. Assimos. Pathophysiology of Urinary tract Obstruction. In: Campbell-Walsh. Urology. $10^{\text {th }}$ edition. Saunders: Philadelphia; 2011. pp. 1087-1121.

9. Yalavarthy $R$, Parikh CR. Congenital renal agenesis: a review. Saudi J Kidney Dis Transpl. 2003; 14:336-341.

10. Woolf AS, Hillman KA. Unilateral renal agenesis and the congenital solitary functioning kidney: developmental, genetic and clinical perspectives. BJU Int. 2007; 99: 17-21.

11. Argueso LR, Ritchey ML, Boyle ETJr, Milliner DS, Bergstralh EJ, Kramer SA. Prognosis of patients with unilateral renal agenesis. Pediatr Nephrol. 1992;6:412-416.

12. González $E$, Gutiérrez $E$, Morales $E$, Hernández $E$, Andres A, Bello l, et al. Factors influencing the progression of renal damage in patients with unilateral renal agenesis and remnant kidney. Kidney Int. 2005;68:263-270.

13. Wissem H, Khaireddine B, Mehdi J, Nebil BS, Tahar MA.Spontaneous Rupture of Renal Artery Aneurysm into the Renal Pelvis: An Unusual Cause of Gross Hematuria and Urinary Retention. Journal of Case Reports. 2013;3:160-164.

14. Consuelo Restrepo de Rovetto, Luz Ángela Urcuqui, Maribel Valencia, Iris de Castaño, Alexander Maximiliano Martínez. Unilateral renal agenesis: case review of ambulatory pediatric nephrology clinics in Cali. Colombia Medica. 2010;41:52-59.

15. Thapa BR, Kumar L, Rao KLN, Parmar $M$. Congenital solitary kidney with obstructive uropathy. The Indian Journal of Pediatrics. 1985;52:205-208. 